\title{
A new crystallization plate for efficient protein crystallization
}

\author{
Da-Chuan Yin ${ }^{1}$ \\ ${ }^{1}$ School Of Life Sciences, Northwestern Polytechnical University, Xi'an, China \\ E-mail: yindc@nwpu.edu.cn
}

In a routine protein crystallization experiment, the first step of protein crystallization is crystallization screening, that is, finding out the chemical or combination of chemicals that can help to crystallize the target protein. During protein crystallization screening process, crystallization plates are often used to hold the droplet array of protein crystallization solutions. There are commercialized crystallization plates, containing mostly 96 wells each plate, compatible to the SBS standard so that the protein crystallization robots can easily handle the plates. In this report, we show a new crystallization plate we call it Cross-Diffusion Microbatch [1] (CDM) plate that is different from the current commercial ones, and the plate showed good performance in protein crystallization screening as well as crystallization optimization.

The CDM plate comprises 96 units corresponding to the 96 conditions of commercial crystallization screening kits. Each unit consists of 4 wells. During crystallization, all 964 wells are sealed to share the same common space. All volatile components in the droplets can freely diffuse in the common space. To compare the crystallization performance we at the same time used a similar crystallization plate (we call it Microbatch plate, or M plate) that resembles the CDM plate except that each of the 96 units is separately sealed from each other during crystallization. Figure 1 shows schematically the geometry of these two crystallization plates.

The crystallization plates exhibit strong flexibility to adopt different crystallization screening strategies. A very promising method is to vary the volume ratio of protein solution to precipitant solution among the 4 wells [2]. Hence, 4 different initial concentrations can be simultaneously used for each precipitant solution, so that more chances of obtaining crystals may be achieved.

We compared the crystallization results with those using commercial sitting drop vapour diffusion (SDVD) plate. It was found that both CDM and M plates are powerful to increase the efficiency to crystallize proteins.

Apart from varying the volume ratios of protein solution to precipitant solution, other strategies, like varying the $\mathrm{pH}$ level in the droplet, can be also used with the new plates. By adding an extra buffer at different pH to the wells separately, we can adjust the final $\mathrm{pH}$ in the crystallization droplet, thus creating different crystallization conditions simultaneously.

We also compared the crystal quality obtained using the CDM plate as compared with those using the traditional SDVD plates, and it was found that the CDM plate can help to obtain better quality protein crystals than the traditional ones[3].

Our study showed that the new CDM crystallization plate may be very useful in practical protein crystallization screening and optimization.

Acknowledgement: This work was supported by National Natural Science Foundation of China (Grant No. U1632126).

[1] Chen, R. Q., et al. (2014). Acta Cryst.D70, 647-657, .

[2] Dong, C., et al.(2016). Cryst. Growth Des. 16, 569-577.

[3] Hou, H., et al. (2015). CrystEngComm, 17, 5365-5371.

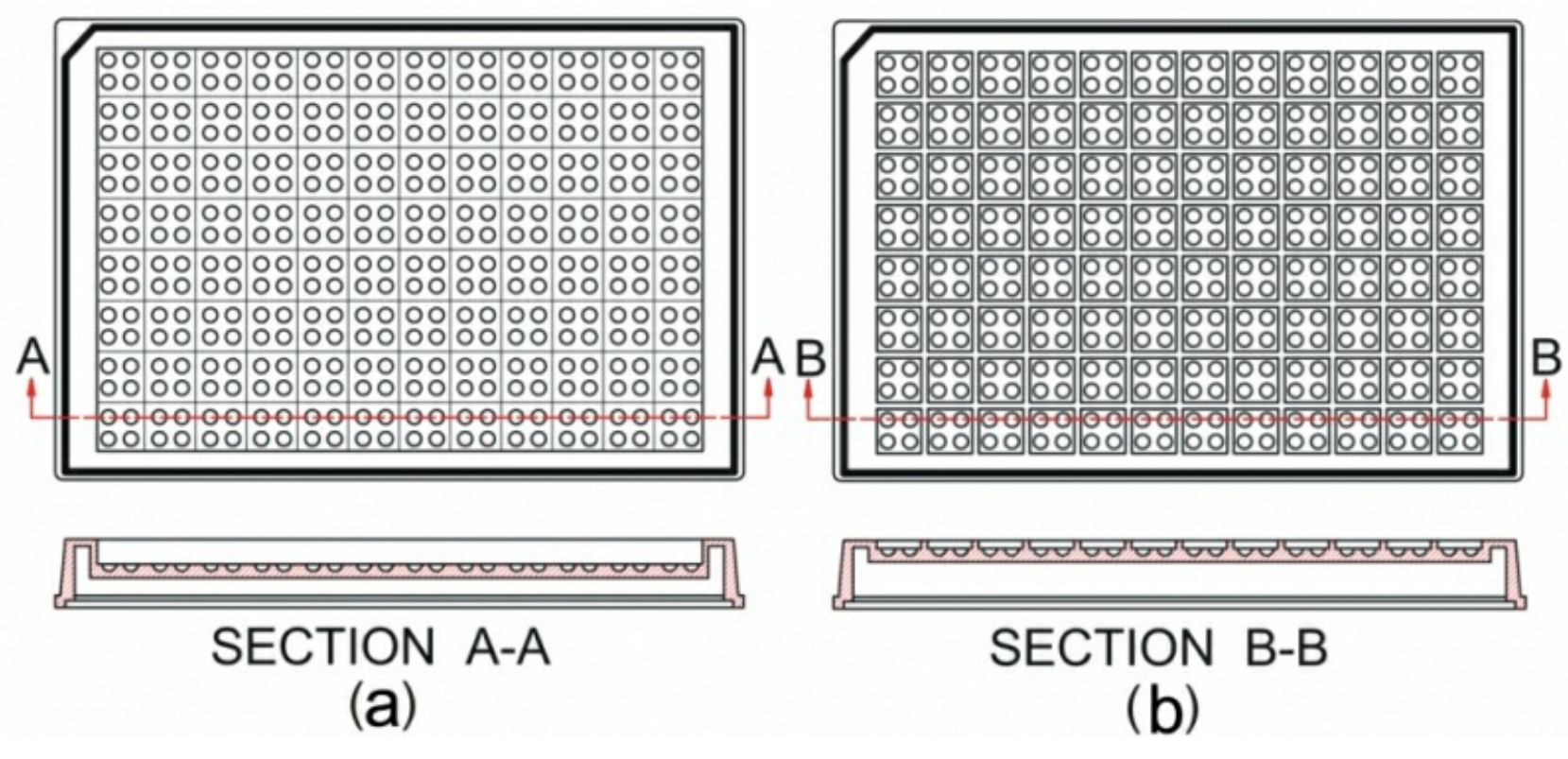

Keywords: protein crystallization, crystallization plate, crystallization screening 